\title{
LUCIEN TESNIÈRE: CRITIQUE ET TRADUCTEUR DE ŽUPANČIČ
}

Lucien Tesnière, d'origine normande, se voua d'abord aux études des langues germaniques pour s'orienter plus tard vers les langues slaves, à savoir le russe, le serbo-croate et le slovène. Dans les années 1921-1924, il fut le premier lecteur de français à l'Université de Ljubljana. Il contribua à la fondation de l'Institut français à Ljubljana et en devint le premier directeur sous la présidence de Oton Župančič. Ses recherches de la langue slovène lui firent parcourir pratiquement tout notre pays; il lia des contacts avec plusieurs intellectuels slovènes. Même après son retour en France où il fut nommé professeur de langues slaves à l'Université de Strasbourg, il maintint des rapports par correspondance, en particulier avec Anton Debeljak, Jože Glonar et Fran Ramovš qui lui fournissaient des renseignements, surtout dans le domaine linguistique. Il entretint un lien encore plus étroit avec Glonar qui lui envoyait les livres récents à ce sujet que Tesnière présentait par la suite dans la Revue des Études Slaves. Au cours de l'année académique 1927/28, il donna un cours de conférences publiques sur la littérature slovène depuis Trubar passant par Prešeren jusqu'à Župančič. ${ }^{1}$

Lors du cinquantième anniversaire de Župančič, au début de l'année 1928, on prépara à Ljubljana toute une série de manifestations auxquelles Tesnière ne pouvait pas participer mais il envoya par contre au poète une lettre écrite en français que nous publions ici en forme intégrale. ${ }^{2}$ Dans cette lettre, Tesnière voit en Župančič un poète slovène de grande qualité et un important interprète de la culture française. Il lui donna des nouvelles sur ses propres conférences tenues à Strasbourg sur Prešeren et sur Župančič.

Cher Monsieur et Ami,

Lorsque j'ai reçu l'autre jour l'invitation à prendre part à la fête qui doit être donnée en l'honneur de votre cinquantième anniversaire, j'ai été partagé entre la joie de voir que la

1 Marko Kranjec: Tesnière Lucien, Slov. biogr. leksikon (Dictionnaire biographique slovène), T. 12 (1980), pp. 66-57. - Jožko Prezelj: L. T. Oton Joupantchitch, Ljublj. zvon 1931, 763- .

2 La lettre est conservée au Département des manuscrits de la Bibliothèque Nationale et Universitaire de Ljubljana. 
Slovénie honorait en vous, comme elle le devait, son plus grand poète, et le regret de ne pouvoir, en raison de la distance, participer à cette belle manifestation.

Mais je ne veux pas laisser passer cette occasion de vous exprimer, tant en mon nom qu'en celui de l'Institut des langues et littératures slaves de la Faculté des lettres de l'Université de Strasbourg que j'ai l'honneur de diriger, l'expression de notre vive admiration pour votre oeuvre slovène et de notre profonde reconnaissance pour votre oeuvre française.

C'est qu'en effet, vous n'êtes pas seulement pour nous dans l'illustre lignée des poètes slovènes l'héritier direct et le continuateur des Vodnik et des Prešeren, mais vous êtes aussi l'incomparable traducteur d'Anatole France et d'Edmond Rostand, le président de notre Institut français et celui dont le génie poétique s'est inspiré de Montmartre-Jérusalem et du parc fermé du Luxembourg.

Ce sont là des titre éclatants à notre reconnaissance, que la France a tenu à marquer en vous faisant entrer dans sa Légion d'honneur.

Mais la vieille affection que $j$ 'ai toujours eue pour le peuple slovène depuis que $j$ 'ai appris à le connaître, ne laisse pas de diriger mes préférences sur votre belle oeuvre en langue slovène. Et, à ce propos je suis heureux de vous faire savoir que je professe cet hiver à l'Université de Strasbourg un cours public, qui est le premier cours de littérature slovène professé en France. J'ai parlé hier de Prešeren, et je crois pouvoir dire qu'à travers les humbles traductions que $\mathrm{j}$ 'ai faites de quelques-unes de ses poésies, le public a vraiment senti et compris votre Pétrarque. Ai-je besoin d'ajouter que ma dernière leçon vous sera consacrée et que ce sera pour moi une joie que de pouvoir dire à mon public la haute admiration et la profonde amitié que j'ai pour vous.

En attendant le jour, trop lointain à mon gré, où un nouveau passage en Slovénie me permettra de vous serrer la main, je vous prie de bien vouloir agréer, cher Monsieur et Ami, avec mes respectueux hommages pour Madame Župančič, l'expression de mes sentiments les meilleurs.

Lucien Tesnière

Peu après, le professeur strasbourgeois envoya à Ljubljana, à Župančič, un article découpé dans un journal français qu'il nous a été impossible d'identifier, donnant un rapport minitieux sur son cours public consacré aux trois poètes de la Moderne slovène et, plus articulièrement à Župančič, évoquant dans sa conclusion la fête récente donnée en l'honneur de ce dernier à Ljubljana. ${ }^{3}$

Plusieurs publications parurent en l'honneur du poète au cours de l'année jubilaire dont l'étude d'Arturo Cronia. ${ }^{4}$ Tesnière qui continuait à présenter régulièrement les nouveaux livres slovènes dans la Revue des Études Slaves, y donna également un court aperçu sur le livre d'Ivo Sever ainsi que sur celui de Cronia: ${ }^{5}$ le livre de Sever qui parut de façon provocative le jour-même de la fête de l'anniversaire du poète avait l'intention de démontrer que Župančič n'était pas un poète de talent mais un plagiaire médiocre. Le livre de Cronia, par contre, lui parut sérieux, le meilleur ouvrage existant sur le poète

3 Cet article est conservé dans le même département de la BNU de Ljubljana.

4 Cronia Arturo: Ottone Župančič. Pubblicazioni dell'Istituto per l'Europe orientale, Prima serie XIII, Roma, Anonima romana editoriale 1928.

5 Revue des études salves 1928, p. 308: Chronique, Publications. Slovène. 
et Tesnière regrette que le ton critique cède si souvent sa place au ton lyrique et que le traité se transforme en simple description. Il interprète ainsi d'une manière erronnée le merveilleux symbole des aurores de la Saint Guy. De plus, 'Zvezna knjigarna' ('La Librairie fédérale') est traduite de façon erronnée par 'Librairie de l'étoile'. "Cela suffit à démontrer que l'auteur possède mal le slovène". Il cite deux années différentes de parution du poème Čaša opojnosti (Une coupe d'ivresse), 1898 et l'autre fois 1899: "Autant de taches qui rendent médiocre un livre qui eût pu être bon."

Les raisons pour lesquelles Tesnière se décida à rédiger lui-même un livre sur Župančič nous paraissent ainsi assez claires. On pourrait considérer les festivités et les publications à l'occasion du cinquantième anniversaire du poète que nous venons de mentionner comme la première d'entre elles. ${ }^{6}$ La deuxième fut le cycle de ses conférences sur la littérature slovène, spécialement la dernière consacrée à la Moderne slovène et à Župančič qu'il avait faites à l'Université de Strasbourg. La troisième raison fut le livre d'Arturo Cronia dont il se réjouit en tant que d'un ouvrage sérieux mais qui le révolta en même temps par ses défauts. La date ajoutée à l'avant-dernier chapitre nous montre que Tesnière écrivit son ouvrage sur Župančič avec application et avec beaucoup de soin de juillet 1928 jusqu'au mars $1930 .^{7}$

L'ouvrage Oton Župančič, poète slovène. L'homme et l'oeuvre est un livre volumineux de presque 400 pages en $16^{\circ}$ qui parut à Paris en $1931 .^{8}$ Cette oeuvre est caractérisée par une construction élaborée, en particulier en ce qui concerne sa double fonction, celle de monographie et d'anthologie. Chacune de ces parties, la monographie d'histoire littéraire aussi bien que l'anthologie des traductions, comprend à peu près une moitié du livre. Dans son Introduction, l'auteur explique lui-même en grande partie les buts et les principes de son travail et de son choix.

Il y constate que Župančič est le plus grand poète slovène de l'époque mais que, comme il s'exprime dans l'idiome d'une petite nation, il est à peu près inconnu dans le monde et aux Français quoiqu'il existe des points communs entre son oeuvre et la littérature française. Ensuite, Tesnière décrit sa première rencontre de Župančič lors de son séjour à Ljubljana, décrivant d'une façon expressive et intéressante les traits caractéristiques de son apparence physique et spirituelle. L'auteur voudrait renseigner le public français sur ce poète important et présenter son oeuvre sommairement sans donner toutefois un travail de littérature comparée qui serait une entreprise risquée. Il voulait surtout faire apparaître l'évolution psychologique et littéraire du poète en analysant ses dispositions d'âme, ses idées et son expression. Cette étude est illustrée et

6 En plus des publications déjà citées, il faut mentionner Jubilejni zbornik za 50-letnico O. Ž. (Hommage à Župančič pour son $50^{\mathrm{e}}$ anniversaire) puisque l'anthologie Naša beseda (Notre parole) ne parut que l'année suivante, rédigée par Fran Albreht.

Tesnière: Oton Joupantchitch - Conclusion, p. 371.

8 Publications de la Faculté des Lettres de Strasbourg, Deuxième série, volume 7. Paris, Les Belles-Lettres 1931. 
complétée par des traductions aussi fidèles que possibles ne respectant toutefois les rimes que de temps à autre. Tesnière ne traduisait que ce qui était le plus important pour les Slovènes, d'une importance particulière pour les Français et traduisible en langue étrangère.

Le chapitre Enfance et adolescence, parle de la première période de création du poète: il souligne le rôle de la poésie populaire dans l'école de Janez Evangelist Krek, il décrit la manière dont Župančič connut Shakespeare et Vienne de la fin de siècle mais ce qu'il ignorait c'est que Župančič avait été actif au sein de Zadruga, société littéraire des lycéens. On rencontre ainsi dans ce chapitre quelques inexactitudes: par exemple l'affirmation que Župančič se soit lié d'amitié avec ses amis Kette et Murn dès son arrivée à Ljubljana par l'intermédaire de Krek tandis qu'en vérité, comme les deux futurs amis n'avaient pas été ses compagnons de classe, il ne les connut que l'un après l'autre dans la Zadruga; puis, le fait d'avoir caractérisé l'almanach Na razstanku ( $A u$ moment de la séparation) de publication collective de la Moderne puisque parmi les quatre amis seul Murn y prit part. Tesnière connaissait très bien les sources disponibles de l'époque et les respectait fidèlement mais il ne pouvait pas connaître l'évolution utltérieure de l'histoire littéraire slovène notamment l'étude importante de Koblar sur la Zadruga. A part l'oeuvre de Župančič, Tesnière suit aussi de très près les événements culturels et politiques de notre pays et renseigne le lecteur étranger sur des faits que l'intellectuel slovène connaît déjà.

Tesnière caractérise le recueil Čaša opojnosti (Une coupe d'ivresse) de lyrisme de la jeunesse. D'après Tesnière, le poète y exprime la volupté et le plaisir épicuriens. L'amour se présente sous différents aspects, sensuel et mystique. Ce que Župančič chante surtout chez les femmes, ce sont leurs yeux. Ensuite, il exprime la mélancolie, la lassitude et la désolation. Il nous présente toute une série de petits tableaux de genre espiègle. Il refuse avec sarcasme les hypocrites et les philistins. Il pèche - dans les Séguedilles, par exemple - gaiement et sans se repentir. Mais sa roža magota (La rose omnipotente) se transforme tristement en roses malades. Enfin, il retrouve le matin serein de son enfance. Les poésies lyriques groupées en sept cycles sont liées par plusieurs romances et ballades. Pour conclure, le poète se moque de ses critiques. Tesnière relie les parties particulières du recueil d'une manière inventive leur donnant des noms lyriques et symboliques. Il interprète le recueil entier d'un point de vue légèrement subjectif, vu qu'il accentue un peu trop le côté sensuel, l'hédonisme ainsi que le caractère blasphémique de Župančič. Pour justifier cette interprétation, il tire des parallèles avec Horace (Carpe diem!), Dehmel aussi bien qu'avec Baudelaire et Verlaine. On pourrait dire qu'il ne souligne pas assez la sombre douleur qui est causée par l'expérience d'un amour malheureux qui n'était pas seulement le reflet d'une expérience personnelle (sa liaison avec Berta, Albertine, par exemple), car on ne put l'expliquer que plus tard après avoir découvert sa correspondance amoureuse de jeunesse. 
Tesnière traduisit 27 des 69 unités formant le recueil Čaša opojnosti (une Coupe d'ivresse) - on peut dire qu'elles témoignent d'une bonne connaissance de notre langue. L'expression de roža mogota inconnue et incompréhensible pour la plupart des Slovènes puisque reprise d'une ancienne légende populaire oubliée originaire de la région de Bovec et de Bohinj, est traduite correctement par rose omnipotente. II reproche à Cronia, qu'il corrige d'ailleurs souvent, que la traduction de l'adjectif dans l'expression jedri udje par eccitante est trop exagérée et il préfère utiliser lui-même le terme de ferme qui lui semble plus approprié. En traduisant les mots abstraits, il change parfois avec beaucoup d'invention le genre des mots: brezmejno okrožje est traduit par l'immensité environnante. Il traduit et comprend certaines poésies jusqu'au moindre détail et avec beaucoup de sensibilité qu'il s'agisse de poésies tendres ou passionnées, tristes ou pleines de douleur, espiègles ou subversives. Pour ses traductions, il ne se sert point des textes de l'anthologie Mlada pota (Les chemins des jeunes) qui ont été remaniés ultérieurement, et adoucis de leur charge émotionnelle mais il se sert de la première version originale de la collection ou même de celle publiée dans une revue. Pour accentuer la force expressive et à cause du rythme, il ajoute souvent au vers originel quelques nouveaux mots. Il essaie parfois d'exprimer l'harmonie à l'aide de rimes mais dans la plupart des cas, il n'y a en a pas dans ses traductions.

Influencé par les décès prématurés et successifs de Kette et de Murn, ainsi que par la position importante qu'occupe le cycle Aux mânes de Josip Murn-Aleksandrov, placé à l'introduction Tesnière appelle trop vite la collection Čez plan (A travers la plaine) Le lyrisme de la douleur. Le titre de ce recueil est également difficile à traduire à partir du cycle mentionné; Župančič utilise en effet souvent l'expression de "polje" ("deux fois "poljana", jamais "plan") pensant à Ljubljansko polje (La plaine de Ljubljana) au-delà de l'ancien cimetière de Saint Christophe. On pourrait voir dans certaines des poésies du II $^{\mathrm{e}}$ groupe de ce recueil d'après leurs thèmes un lien avec Baudelaire et Dehmel. La comparaison de Druga belokranjska (La deuxième blanc-carniolaise) que Tesnière relie au cycle serbe sur le roi Marko, avec pour seul trait commun le nom du jeune homme, et l'explication de Moravska narodna (Chanson populaire morave) où Župančič exprime le regret de ne pas avoir joui plus de la vie au sens épicurien, nous semblent inappropriées. Par contre, Tesnière relie avec succès le cycle d'introduction sur Murn et (presque) la poésie finale Ptič samoživ (L'oiseau de vie) pour leur idée commune sur la douleur du poète, sa solitude et son courage. Il relève dans ce recueil sur le rapport du poète avec la nature et avec la patrie

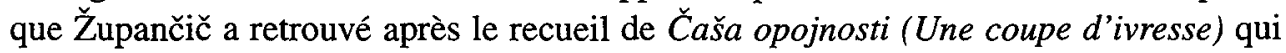
était assez artificielle et individualiste.

Il est néanmoins intéressant de constater qu'il n'ait pas inclu dans son choix ni analysé du point de vue idéologique des poésies comme Meni se hoče ( $J$ 'en ai envie), Pesem mladine (La chanson des jeunes) Pokopališče sv. Barbare (Le cimetière de Sainte Barbara), Daj, drug, zapoj (Vas-y, compagnon, chante!) qui avec quelques autres poésies y inclues, telle que Vseh živih dan (Le jour des vivants) et Raznim poetom ( $A$ 
divers poètes) confèrent à ce recueil (y compris son titre) un caractère idéologique tout autre de celui que lui est attribué par Tesnière - à savoir le caractère de la volonté, du courage, de la vitalité et de l'optimisme.

Tesnière a traduit 33 des 67 unités contenues dans le recueil Čez plan (A travers la plaine), c'est-à-dire la moitié, tandis que les cycles Manom Aux mânes de Joseph Mourn-Aleksandrov et Jutro (Le matin), eux, ont été traduits intégralement. Dans le premier cycle, le poème le plus connu (le condor prisonnier) dans son ensemble est traduit avec soin et sensibilité, à part trois points qui sont: dans la $5^{\mathrm{e}}$ strophe parlant de pest-meč (poing-épée), il ne réussit pas aussi bien que Župančič à reproduire par la rime intérieure de "e" de meč-žareč (épée-brûlant) et par la répétition des "o" la mélodie de l'original; dans la dernière partie de la poésie, il traduit dva črna curka par deux noirs jets de larmes au lieu de "... de sang" puisque le condor se heurtant sans succès contre le grillage "saignait"; un peu plus loin, Tesnière omet par mégarde le refrain veř, ravno čez oči - dva črna curka (oui, de ses yeux deux noirs, noirs jets de larmes)! Dans la deuxième poésie du cycle, à deux emplacements où Župančič décrit plus longuement le regard et la barbe de son ami décédé, on déplore l'absence de musique si riche et suggestive, dans l'original créé par les rimes intérieures ainsi que par les consonnes et les voyelles $\check{c}, o, a, o$ retraçant bien l'atmosphère. Le traducteur ne réussit que rarement à reproduire des rimes extérieures. Il s'agit, il est vrai, de finesses qu'un traducteur, qui n'est pas poète lui-même, réussit difficilement. Dans la septième poésie, l'avantdernière du cycle, l'expression de Tesnière mon temple pour moj hram est exagérée puisqu'il s'agit ici du sens de "maison" et non de "sanctuaire".

L'expression "le petit vin de Karst" pour le vin "teran" dans le poème Vseh živih dan (Le jour des vivants) probablement ne correspond pas complètement à ce vin puisqu'il ne s'agit pas d'un petit vin léger. Dans la deuxième strophe du même poème, Tesnière encore une fois, ne réussit pas à reproduire phonétiquement l'orage aussi bien que Župančič, qui à cet effet a employé de nombreux "r" bruyants ainsi que de nombreuses rimes intérieures et extérieures. Le mot dialectal rdeči nagrlin dans la Belokranjska (Chanson blanc-carniolaise) n'est employé qu'en tant que forme littéraire pour "l'oeillet" et ne peut pas être traduit par le bluet. Dans le poème Slovesu (Adieu), ne comprenant qu'une sule strophe, les langues ont suggéré au poète et au traducteur les jeux de mots videti/vedeti, voir/ savoir. Ailleurs, dans le poème Doma (A la maison), le texte original se prête très bien à la traduction: To je sèn, to je laž-C'est un songe, c'est un mensonge! et fait apparaître un jeu de mots qui n'existait pas dans la version slovène. Dans le poème rappellant Baudelaire, Čez ves obraz (Ses cheveux sont tombés par devant son visage) auquel Tesnière tenait particulièrement, il s'efforce exceptionnellement à donner les rimes finales à tous les vers. Dans Japonski motiv (Le motif japonais), Tesnière traduit littéralement notre dicton $A$ želje gredo mi $v$ vas / $k$ srčno ljubljeni devici par Mais mes désirs vont au village alors qu'on devrait avoir Mais mes désirs vont voir... Dans la Moravska narodna (Chanson populaire morave) qui est tout à fait remaniée on ressent dans la version originale les vers časi, mladi časi, / neužiti 
krasi! comme nostalgiques, tendres, tandis que dans la traduction de Tesnière relève une note sensiblement sensuelle: $O$, temps de ma jeunesse, / charmes non déflorés!

Le cycle Dan (Jour) est le plus révélateur quant aux caractéristiques de Tesnière dans le domaine de la traduction: il maîtrise à la perfection la langue de l'original, il capte chaque fois le ton de la poésie, il essaie de reproduire le style de l'original mais ses faiblesses apparaissent dans le domaine musical - le cycle reste sans rimes finales. Dans le poème Ob Kvarneru (Sur le Quarnero), il traduit assez librement na vrhu klanca par en haut du sentier, il traduit par contre d'une manière exacte quoique plus descriptive un autre vers bien plus difficile vzlepetalih ptic - des oiseaux qui bruissent dans les airs. Il est intéressant de constater que dans le poème Ptič samoživ, il traduit maladroitement par un. substantif L'oiseau de vie alors qu'au début de la troisième strophe, la traduction est bien meilleure, Carc'est l'oiseau qui puise en lui-même la vie.

Le recueil Samogovori (Les Monologues) est très bien déterminé par l'auteur de cette monographie sous le titre de Le lyrisme de la méditation. Il dit d'abord quelques mots sur les années d'études du poète à Vienne, sur son séjour à Paris et sur la conception des Samogovori (les Monologues). Il intitule les différents cycles du recueil la mélancolie, le sarcasme, religieux, celui où libéré du conflit par ses méditations, il retrouve la sérénité suprême, et celui de la patrie. La mélancolie lui est inspirée par le printemps, la femme et les ombres de la nuit; dans ce cycle, Tesnière est attiré surtout par la poésie Zaprti park (Parc fermé), qui fut écrite à Paris, et qui lui rappelle un peu Verlaine. Dans le cycle des poésies sarcastiques, il cite l'exemple de Vesela pomladna epistola (Joyeuse épître printanière) dont le contenu n'est pas orienté contre le patriotisme autrichien mais bien contre le faux amour de la patrie slovène. Dans le cycle religieux, Tesnière préfère analyser un nouveau motif parisien: Pogled na Montmartre (En contemplant Monmartre) qui lui semble symboliser la consolation pour la souffrance endurée par les hommes et Tesnière enchaîne avec le discours sur la recherche de Dieu, évoquant son conflit intime tranchant, sa confiance en la vie et en lui-même. Le cycle où le poète se sent libéré par ses méditations est présenté par Tesnière dans la poésie Ribnik (L'étang) qui chante le reflet dans l'eau, l'amour de la dualité des choses et le calme de la vie. Il laisse de côté les deux poésies les plus importantes de ce cycle, Prebujenje (Réveil) et Nočni psalm (Psalme de la nuit) où Župančič trouve un moyen de se libérer de la solitude, du conflit intérieur et du désespoir au sein de la communauté des hommes et du pantéisme spirituel. Tout en analysant avec soin et avec certain succès les thèmes et les idées de la poésie lyrique de Župančič de cette période, l'auteur de la monographie ne prend que rarement en considération son style et sa forme, particulièrement raffinés dans les Samogovori (Monologues).

Dans le cycle consacré à l'amour de la patrie, Tesnière ne fait qu'un court aperçu de la poésie $Z$ vlakom (Avec le train) affirmqant qu'il s'agit d'un voyage allant de Ljubljana à Trieste alors qu'en vérité, Župančič y décrit le trajet de Ljubljana, à Vienne, passant par la Štajerska: le champ de Ljubljana, la Šmarna gora (Montagne de Sainte 
Marie) et les Montagnes de Kamnik ne peuvent être vues si le train prend la direction vers Trieste. Un peu comme dans le cycle Manom Josipa Murna-Aleksandrova (Aux mânes de Joseph Mourn-Aleksandrov), Tesnière s'arrête assez longuement sur la poésie Duma (La Douma) où Župančič chante la beauté de la terre slovène, celle de Bela krajina et surtout le grand monde, son progrès universel et la grande émigration à l'étranger. Il compare souvent le thème de cosmopolitisme et le sytle élevé de Župančič à celui de Whitman et de Verhaeren sans souligner assez la composition évidente de Duma (La Douma): la voix féminine, la voix masculine et le coeur du poète; le caractère patriarcal de notre campagne - le progrès dans le monde; les souvenirs d'enfance - la coscience amère de l'émigration. Duma (La Douma) ouvre à Tesnière le chemin vers les thèmes patriotiques présents aussi dans le recueil de $V$ zarje Vidove (Aux aurores de la Saint-Guy).

Parmi les 46 poèmes du recueil de Samogovori (les Monologues) il en choisit 23, c'est-à-dire juste la moitié et Duma (La Douma) complet. Dans la traduction de la poésie $\mathrm{Na}$ hribu (L'homme sur la colline) on aperçoit de nouveau l'un des principaux défauts de Tesnière-traducteur: l'absence du sentiment de la musique dans les rimes devant le refrain "kam"? (où?). Cette impuissance quant à la reproduction des sentiments, du style et aussi bien de la musique du poète se retrouve aussi bien dans la traduction des autres poésies du recueil. On s'en rend compte surtout dans le poème Umetnik in ženska (L'artiste et la femme) en comparant les deux vers slovènes avec leur traduction ne recréant point la petite musique intime trouvée chez Župančič: Nocoj/ zvenijo zvezde zopet kot nekdaj - Cette nuit/ on entend à nouveau, comme jadis, l'harmonie des étoiles. Dans la poésie Melanholija (Mélancolie) le traducteur fait exceptionnellement des rimes presque à tous les distiques mais point à tous. Il interprète avec une attention particulière et un amour spécial le motif triste et rêveur de Zaprti park (Le parc fermé) et celui de la poésie Tiho prihaja mrak (Doucement l'ombre arrive) dont la traduction est très ressentie et réussie.

Tesnière traduit avec le même zèle le motif parisien suivant, Pogled na Montmartre (En contemplant Montmartre) qui est relié au souvenir du saint sépulcre tel que le poète l'a gardé de sa jeunesse. Il traduit avec le même soin et d'une manière efficace les autres poésies philosophiques difficiles soit par leur réflexion soit par leur expression. Néanmoins, le traducteur aurait dû changer, vers la fin de la troisième strophe de la poésie Klic noči (L'appel de la nuit) l'adjectif possessif de la troisième personne du pluriel ses pieds pour l'adjectif possessif de la première personne du singulier, mes pieds. Dans la poésie Samogovor (Monologue), on trouve une inexactitude dans la huitième strophe: $\mathrm{Pa}$ rad bi [imel] očetovo hišo spet/ s hinavščino - Et tu voudrais remplir encore la masion de ton père/ de ton hypocrisie (qui fut légèrement changé pour l'édition des Dela (les Oeuvres) I, 1936 par Župančič en: Pa bi rad v očetovo hišo spet...). Dans la poésie Sebi (A moi-même), Tesnière n'a apparemment pas compris l'expression littéraire vieillie "mladoletje" signifiant "le printemps" puisqu'on retrouve 
dans la traduction "jeunesse": Kličeš, drevo, mladoletja? - Arbre, est-ce ton adolescence que tu appelles?

Vue que la poésie lyrique de Župančič est la plus exigeante et la plus perfectionnée du point de vue forme dans le recueil de Samogovori (Les monologues), permettez-moi de faire un court aperçu de sa forme dans la traduction de Tesnière. Les poésies qui sont en forme de strophes dans l'original, et c'est le cas de la majorité - seuls sept poèmes, les plus larges sont écrits en vers libre, parmi lesquels on trouve Duma - conservent la même division et la même forme en strophes dans la traduction. Le vers dans la traduction de Tesnière a presque toujours quelques syllabes en plus mais le traducteur s'applique à reproduire le plus fidèlement possible le rythme de l'original. Les rimes qui dans la version slovène sont particulièrement riches et compliquées, ne se retrouvent que rarement voire pas du tout comme dans les poésies intégrales ni fonctionnant alors plutôt comme une décoration musicale fortuite et non comme un moyen musical en harmonie avec le sujet.

Dans le cycle de la patrie, la traduction de la poésie $Z$ vlakom (Avec le train) $\mathrm{s}$ 'approche beaucoup de l'original aussi bien du point de vue thème que style, où le poète montre son enthousiasme et son admiration pour la terre slovène, où il en déclare son amour. La traduction de Duma (La Douma) excelle encore plus probablement à cause de sa forme en vers libre. On peut difficilement croire qu'un étranger, en l'occurence Tesnière quoique polyglotte de talent, puisse posséder jusqu'à la perfection à quelques détails près le vocabulaire slovène pour reproduire aussi brillament le style et le rythme du poète à l'aide des plysyndètes et d'anaphores. Toutefois, il ne réussit pas toujours à reproduire les onomatopées et la musique à certains endroits dans la poésie, ${ }^{9}$ notamment dans la plainte de la veuve ou dans la sonnerie de la cloche où le poète fait alterner les voyelles $o$ et $\mathrm{i}$ avec des consonnes dures, créant nombreuses rimes intérieures ou extérieures. Quoique la traduction de Duma (la Douma) en général soit excellente, on peut y trouver quelques endroits délicats, des fautes et des imperfections dans l'expression. Tesnière est parfois trop descriptif et maladroit: [kiparjevo dleto] s skale narahlo je snov odpoljubljalo - du roc délicatement ses baisers [du ciseau du sculpteur] tiraient la forme; škrjanček ... je pesmi pršil - l'alouette ... a laissé tomber son chant en fine gouttelettes. A d'autres endroits, Tesnière hésite quant à la signification de certains mots: la question de Župančič In čuješ na tnalu trlice? est traduite par: Et entends-tu le broiement du chanvre?. Le terme de la Blanche Carniole tnalo dans le sens de "la cour" y est omis terme que d'ailleurs la majorité des Slovènes ne connaît pas non plus et que le poète lui-même nota quelque part accompagné de

9 Hrasti, orjaki... se z burjo borijo; ti pa se mučiš... in dušo dušiš; moj ponos se pne in pesem z njim; ritem rok in ramen; zmotnim mrmranjem vesti spremljajoč; škrjanček - pojoča raketa - je pesmi pršil. Ces vers sont traduits par Tesnière en: Les chênes, des colosses.... luttant avec la tempête; et toi, tu peines... et tu étouffe tom âme; voici mon orgueil qui se redresse, et avec lui mon chant; le rythme des bras et des épaules; accompagnant d'un grognement terne les nouvelles; l'alouette - une fusée qui chante - a laissé tomber son chant en fines gouttelettes. 
l'explication: "tnalo: l'espace devant la maison s'il n'est pas limité par une clôture." Un autre exemple relevant du vocabulaire, est la phrase "se ptički za šolskim vrtom v mejici gostili so" où Tesnière traduit le mot mejica traduit par le sillon, alors qu'il est évident qu'il s'agisse de la haie et précisément de la haie de buissons de charme, fait confirmé un peu plus loin dans la poésie. Il faut mentionner que dans la traduction de Duma (la Douma), il manque un vers, razkladal skrivnosti glasov in družine dreves qui serait peut-être dû au manque de place lors de la mise en page.

De même que pour le recueil de Samogovori (les Monologues) il convenait de le qualifier de lyrisme de la méditation, ainsi le recueil suivant, $V$ zarje Vidove (Aux aurores de la Saint Guy) est très bien caractérisé par Le lyrisme de la patrie. Dès le début, Tesnière refuse l'explication de Cronia qui affirmait que le titre était lié à un des événements de l'histoire serbe et à l'ancienne fête nationale et influencé surtout par Glonar, $^{10}$ il donne une explication correcte du titre le reliant au solstice d'été, c'est-à-dire au jour le plus long de l'année, qui pour Župančič représente le symbole de l'apogée de l'histoire du peuple slovène. Ensuite, Tesnière passe en revue le passé historique récent des Slovènes commençant par l'année décisive de 1908 pour continuer avec la Première Guerre mondiale en concluant avec les injustes traités de paix. Il est très critique face aux alliés occidentaux puisque, lors du plébiscite carinthien, dit-il, la France se montra faible comme toujours, l'Angleterre se montra perfide, l'Italie se montra imprudente et l'Amérique ne se montra point ce qui eut pour seul effet le succès de la politique de germanisation exercée par l'ancienne Autriche et le sanctionnement des résultats par le droit de la violence et de l'injustice des siècles passés. ${ }^{11}$ Tesnière donne à ses compatriotes un exemple celui de l'état d'esprit qui régnerait en France si on avait attribué à l'Angleterre et à l'Espagne un tiers de leur pays comme cela avait été fait pour les Slovènes.

En passant à l'explication du recueil, Tesnière se trompe en supposant que la poésie d'introduction, Slap (La cascade) chantant l'écoulement de la nature puissante et de l'homme, fut inspirée au poète par la cascade de Savica près de Bohinj; il s'agit en vérité de la cascade de Peričnik à Bled car, la poésie fut écrite peu après le mariage du poète et il y parle de son séjour avec sa jeune fiancée dans la villa des Kessler et de leur excursion dans la vallée de Vrata et à la cascade. D'après Tesnière, les trois cycles du recueil chantent le passé imminent, le présent et le futur de la nation du poète. Dans le premier cycle, Dies irae annonce la terrible guerre mondiale; la chanson prolétarienne Žebljarska (Chant des cloutiers) représente pour Tesnière "un chef-d'ouvre d'harmonie imitative" tandis que Glad (La faim) personnifie la misère et l'abrutissement; troils poésies raccontent la souffrance d'un père au front telle que ses enfants la perçoivent; une d'elles parle des réfugiées, de la mère des Brda et de la Sainte-Marie de Sveta gora. Dans le deuxième cycle, le poète exprime dans sa poésie Podobi (L'image) son 
aspiration et celle de sa patrie à acquérir une image intérieure vraie et harmonieuse; dans la poésie Zemljevid (Carte de géographie) le poète exprime son pressentiment et son appréhension de voir les frontières de la Slovénie devenir le butin des impéralismes; dans Kovaška (Chant des forgerons), il passe en revue les couches sociales qui devraient chacune choisir un leader national - une occasion pour Tesnière de comparer de nouveau Župančič avec Whitman, tandis que Naša beseda (Notre verbe) en tant qu'hymne du peuple libéré représente selon lui le point culminant du recueil. Dans le troisième cycle, celui de la maturation et de la fécondité, le poète explique notre responsabilité dans les rapports amoureux devant notre nation (Telesa naša (Nos corps), Mrtva nevesta (La fiancée morte), Pričakovanja (Attente), consacrant le recueil à sa femme. Ces considérations sérieuses du poète dans le domaine de l'amour représentent en fait l'élément essentiel de ce recueil où Tesnière pourtant omet de mentionner une autre qualité importante: le panthéisme spirituel que le poète ressent au bord du Lac de Bled Jezero (Le lac), Tih, tih je kraj... (Qu'il est silencieux cet endroit), Zlata jutra (Les matinées dorées). Dans la dernière poésie, Mesečina (Clair de lune), Župančič reproduit l'atmosphère et l'état d'âme symboliques des Vidove zarje (Aux aurores de la Saint-Guy), du solstice d'été, reprenant au début et à la fin de ce poème l'image de la cascade, de glace au début libérée et ruisselante à la fin. En plus de la liberté nationale, cette poésie renferme également l'idée du panthéisme spirituel: le coeur qui est au milieu symbolise Janez Evangelist Krek, alors à la tête du mouvement de la libération nationale, alors que l'Esprit-oiseleur représente le Créateur mystérieux de l'univers.

Župančič lui-même considérait ses premiers trois recueils de façon plus ou moins critique et celui qu'il appréciait le plus était celui de Zarje Vidove (Aux aurores de la Saint-Guy). Tesnière choisit dans ce recueil 16 parmi les 32 unités, à savoir ceux qui se prêtaient le meiux à la traduction, c'est-à-dire la moitié, comme dans les Monologues. Il prit le premier cycle presque dans sa totalité; dans le deuxième, il omit ceux traitant de thèmes politiques spécifiquement slovènes ceux d'un accès plus difficile pour les étrangers; du troisième, il omit quelques poésies d'amour, la poésie Vihar (L'orage), trop riche par sa musique, et quelques poésies méditatives surtout celles à caractère panthéiste que nous venons de mentionner.

Dans ce recueil aussi, le point le plus faible dans la traduction de Tesnière se retrouve dans son interprétation de la musique. Dans la poésie Slap (La cascade), il essaya de reproduire la sonorité du refrain à l'aide des assonances à la fin des vers. Il réussit mieux à reproduire l'horreur que suscite la grande cloche dans Dies irae par le choix de certaines voyelles et consonnantes le tout restant toutefois sans rimes; son interprétation musicale des chevaliers apocalyptiques et de l'orgue assourdissant est moins réussie quoique ce texte expressionniste et difficile à traduire soit dans son ensemble un texte remarquable. Dans la traduction de Žebljarska (Chant des cloutiers), la fonction musicale est conservée seulement dans le refrain tandis que les sons représentant le soufflet de forges et les clous, parmi lesquels des sons qui n'existent pas en français, comme par exemple $\check{c}$, ne pouvaient pas être reproduits. Dans la poésie 
Glad (La faim), Tesnière traduit assez bien le vers plein d'effets sonores čuj žvižge, topòt ob trdi tlak par écoute ces sifflements, ce piétinement sur le dur pavé!

Ne connaissant point les coutumes de la Carniole Blanche, il traduit le vers Brez svetega Duha je strop... par Il n'y a pas de Saint-Esprit sur le toit (au lieu de sous le plafond). Trois thèmes de guerre Otroci molijo (Les enfants prient), Razgovor (Conversation) Dete čeblja (L'enfant gazouille) ne posèrent point de problèmes phonétiques à Tesnière mais il fallait y trouver des expressions reflétant l'état d'esprit enfantin, c'est-à-dire entrer dans l'âme d'un enfant ce qu'il réussit très bien dans cette poésie ainsi que dans celle de Begunka pri zibeli (La fugitive au berceau).

Tesnière traduit moins bien la poésie Podoba (L'image) mais il réussit celle de Zemljevid (Carte de géographie). La poésie en vers libre, Kovaška (Chant des forgerons) quoique transmise par Tesnière au lecteur non-slovène par pleusieurs exemples et acompagnée d'un riche commentaire, contient quelques erreurs: le passage mizarji šentviški, Solkanci, /drvarji po šumah devrait être traduit par menusiers de Chent Vid, de Solkan, Bûcherons des forêts... et non menusiers de Chent Vid, bûcherons des forêts de Solkan..., puisque les habitants de Solkan ainsi que ceux de Šentvid étaient renommés comme étant de bons menusiers, n'exerçant pas le métier de bûcheron; Kapla (Železna Kapla) ne se trouve pas en Styrie Inférieure mais en Carinthie il ne s'agit pas non plus de Šentvid dans la vallée de Vipava mais de Šentvid près de Ljubljana. La poésie Naša beseda (Notre verbe) si difficile à traduire connut-elle une excellente traduction mais il aurait fallu tout de même mettre une note pour signaler au lecteur non slovène que, dans le vers Bil je med nami mož kot zrno klen in zdrav - Il y avait parmi nous un homme robuste et sain, il s'agit de Janez Evangelist Krek; l'adverbe slovène nenehoma, à la fin de la deuxième strophe, fut échangé par mégarde et à cause de sa ressemblance extérieure avec nenadoma et traduite par Tesnière comme brusquement, alors que le vers musical Vej, vrli vejavec, vihar! fut très bien traduit par Vanne, brave vanneur, vanne, ouragan!

Dans la poésie Telesa naša (Nos corps) Tesnière n'a pas bien compris le vers in vkup nas biča [volja bodočnosti], ženo in moža le traduisant par elle jette homme et femme aux bras l'un de l'autre puisque le verbe bičati ne peut être traduit que par fouetter. La poésie Mrtva nevesta (La fiancée morte) est transmise en français avec beaucoup de sentiment encore renforcé dans Pričakovanje (Attente) où dû à une faute typographique, un vers est omis, à savoir: med praprotjo, med gosto mrežo vej (dans la fougère, au bras des branches entrelacées). La poésie finale, Mesečina (Clair de lune), une des poésies les plus profondes de Župančič, fut merveilleusement traduite par Tesnière à deux remarques près: dans le vers iz tvojega srca, iz tvoje postave dans la $6^{\mathrm{e}}$ strophe, le dernier mot ne signifie pas le corps mais la loi c'est pourquoi Tesnière aurait dû mettre ta loi à la place de ta personne; dans la traduction du vers suivant, kar je, se rodi in rodeč te razširja le pronom est en personne erronnée: et s'élargit en efantant à la place du correcte: et t'élargit en enfantant. 
Dans sa monographie consacré à Župančič, Tesnière ne s'est pas limité seulement à l'analyse de la poésie du poète qui représente en effet la partie essentielle et la plus importante de son oeuvre mais il orienta son attention aussi bien à d'autres domaines de sa création, surtout dans le chapitre L'essor dramatique où il analyse d'un point de vue critique l'oeuvre du poète et il parle largement, avec précision, correctement et d'une façon approfondie de Veronika Deseniška (Véronique de Dessentitse). D'abord, il donne un court aperçu de l'histoire des comtes de Celje et des auteurs qui se servirent jusqu'alors de cette thématique dans leurs oeuvres littéraires. Ensuite, il donne le contenu des cinq actes de la tragédie de Župančič et l'analyse des caractères des quatre personnages principaux où il constate que les caractères de Véronique et de Frédéric sont assez contradictoires alors que ceux de Herman et de Jelisava sont plus cohérents. Selon ses propres mots dans Pravdač (Défenseur), le poète voulait dans Veronika Deseniška exprimer la naissance de l'âme slovène tandis que, dans les comtes de Celje, on doit y voir les représentants typiques de féodaux allemands. D'ailleurs, la tragédie de Župančič n'est pas tellement historique mais plutôt psychologique. Il s'agit d'une tragédie d'amour fatale, élémentaire et irrésistible qui suscite en nous une compassion pour Véronique selon la définition sur la culpabilité tragique d'Aristote. D'après sa construction et sa technique, cette pièce est plutôt lyrique que dramatique, l'action se déroule lentement ou il fait même défaut, il s'agit en fait d'une suite de symboles rappelant stylistiquement l'oeuvre de Maeterlinck, plus appropriée pour la lecture que pour le théâtre. Pour terminer, Tesnière parle des critiques de cette tragédie: il refuse d'abord Josip Vidmar pour avoir été trop négatif quoique lucide, ensuite le rédacteur France Koblar pour n'avoir mis que des remarques de caractère religieux, c'est-à-dire non littéraires, et finit avec le livre de Ivo Sever qu'il désigne comme "un factum dont le ton est celui de la polémique". Il conclut le chapitre avec les projets de Župančič (drames historiques relatant l'histoire des Slovènes) en espérant qu'il pourra les réaliser.

Le choix des scènes à traduire par Tesnière est très rationnel: l'introduction (I/2 Plašč (Le manteau); l'amour individuel (III/3 et 5 - Veronikino priznanje (La confession de Véronique), Friderikova vrnitev (Le retour de Frédéric) et la politique d'état (IV/7 et 15-17 - Smrt Hermana mlajšega (La mort d'Hermann) Celjski sèn (Le rêve de Tsélié); la conclusion (V/1 - Veronikin samogovor v ječi (Monologues de Véronique). La traduction, elle-aussi, est tout comme la partie monographique munie quoique modestement, de notes et des indications des sources. Tesnière traduit avec facilité et avec beaucoup de sensibilité.

Dans la scène d'introduction, il faut comprendre la fin de la réponse de Sida Desenice so za njó comme Veronique a dépassé Dessenitsé et pas comme elle est l'héritière de Dessénitse comme Tesnière l'avait traduit. ${ }^{12}$ Desničan (Le châtelain dans la traduction) introduit la phrase d'Hermann dans son récit de la bataille avec les 
Prusses: Ti Deseničan, zvračaš nam bokale,/ kakor si Pruse; pour le verbe à deux sens "zvračaš" du poète, Tesnière fait correspondre avec invention deux verbes presque homonymes: Tu sables le bon vin comme tu sabres les Prussiens. ${ }^{13}$ Dans le même récit du Châtelain, on trouve aussi la phrase suivante: Celjan zdaj več ne séde zraven - vraga où zraven vraga ne signifie pas à côté du diable mais est une exclamation-juron ajouté et par conséquant la traduction Le comte de Tsélié ne peut plus s'asseoir auprès d'unpauvre diable ${ }^{14} \mathrm{n}$ 'est pas exacte.

Vers la fin de la scène du retour de Frédéric au troisième acte, Iélissava désespérée s'exclame: Tla, ste še trdna pod menoj? Ste trda? Tesnière ne reprend pas les deux adjectifs slovènes se ressemblant il est vrai, phonétiquement mais différents de signification, et les traduisit qu'approxamitivement: Pourquoi le sol est-il si dur sous ma personne? ${ }^{15}$

Dans la scène du rêve de Celje dans le quatrième acte, Hermann avertit Véronique qu'il pourrait être encore plus sévère quand il souffre de la goutte: prekleti moj podgrom - da bi ga vrag... et peu après: spet ga v kolenih čutim: žal vam bo. Dans la traduction de ces deux passages, on s'aperçoit de la différence du genre faite par Tesnière, probablement sous l'influence du genre en slovène: ah, ma maudite goutte... je le sens à nouveau dans mes genoux. ${ }^{16}$ Lorsque Hermannn apprend la mort de son deuxième fils, et que Frédéric, provoqué, déclare présomptueusement qu'il est devenu le seul héritier, le chevalier Jošt avertit ce dernier de ne pas se précipiter: Nikar. Ne veš, kako je sklenjeno! Cette expression impersonnelle à la $3^{\mathrm{e}}$ personne du singulier chez Župančič fait supposer qu'il parle du destin inconnu, de ce fait la traduction de Tesnière à la première personne du pluriel est fort douteuse: Pas un mot, Frédéric. Tu sais ce que nous avons décidé! ${ }^{17}$ Lorsque Hermann accepte que Véronique soit la femme de Frédéric à la cour de Celje mais pas pour la vie publique, Frédéric, humilié, dit à son père: Za norca naju imaš. Nimaš pravice,/ da si mi oče., Tesnière ne traduit pas cette phrase très fidèlement: il l'adoucit en partie: Tu te moques de nous. Tu n'en as pas le droit, / bien que tu sois mon père. ${ }^{18}$

Tesnière a une excellente connaissance du vocabulaire slovène; la traduction correcte de l'expression ozimina par les semailles d'automne dans le passage du $\mathrm{V}^{\mathrm{e}}$ acte de Veronika Deseniška n'en est qu'une preuve de plus.

Tesnière caractérise le chapitre suivant: Jerala (Iérala), Une ébauche épique d'épopée philosophique satirique restée inachevée revélant les contrastes entre un

\footnotetext{
13 Ibidem, p. 254.

14 Ibidem, p. 255.

15 Ibidem, p. 270.

16 Ibidem, p. 280.

17 Ibidem, p. 289.

18 Ibidem, p. 293.
} 
bourgeois et un artiste slovènes, entre la matière abrutie et l'esprit agile permettant d'y voir ainsi une certaine ressemblance avec Fauste de Goethe. En un quart de siècle, Župančič n'écrit et publie que quatre "chapitres" dont Tesnière ne mentionne que le Trosième. ${ }^{19}$ Župančič situe l'intrigue dans le Karst. Les courants des eaux souterraines symbolisent la création poétique; le pluriel des noms des rivières auraient été empruntés aux poètes français. Il est inutile de situer ce chant (de Jerala concrètement) dans les grottes de Postojna puisqu'il s'agit ici du karst slovène en général.

Le cant choisi est merveilleusement traduit à une exception près: l'effet mélodieux des rimes à la fin des vers et l'alternance des voyelles et de la consonnante $\check{c}$ dans les verbes se mučijo, točijo, jočejo, mečejo, kličejo ne se retrouvent pas en français. Tesnière omet le vers $v$ neznanska brezna se mečejo.

Tesnière attribue une grande attention au cycle de la Poésie pour enfants. L'âme du poète est d'une certaine manière toute proche de celle des enfants, c'est un pédagogue avec beaucoup de tact et un sens d'esthétique remarquable. Ces poésies trouvent souvent leur place dans les livres de lecture, démontrant ainsi que la poésie pour enfants peut renfermer les éléments de la vraie littérature. En 1900, Župančič publia ses poésies pour enfants qu'il composa dans sa jeunesse, en 1913 dans les années après son mariage, selon Tesnière, trois recueils suivants auraient dû paraître. Tesnière se trompe puisque les poésies du recueil Lahkih nog naokrog (D'un pied léger dansons en rond) furent écrites déjà en été 1908 et parurent vers la fin de 1912 et non en 1913 comme l'affirmait le bibliographe Janko Šlebinger. ${ }^{20}$

Ensuite, Tesnière parle de Pisanice (Oeufs de Pâques), de la tradition populaire des oeufs de Pâques et de la grande variété des poésies dans ce recueil: des motifs petit-russes et surtout ceux évoquant la Blanche Carniole, des thèmes originaux tristes, des ballades dont certaines sont humouristiques et plaisantes, dont le rythme nous rappelle celui de Levstik.

Tesnière désigne à juste titre le recueil Lahkih nog naokrog (D'un pied léger dansons en rond) d'album à images pusiqu'il s'agit d'un livre d'images. Mais ce qu'il ignorait c'est quil s'inspira des poésies allemandes pour écrire ces poésies et que ces images étaient des reproductions allemandes. Župančič n'aimait ni les unes ni les autres et les élabora à sa manière et à tel point qu'elles furent considérées à juste titre comme siennes. Tesnière mentionne plus particulièrement Kraljevič Marko in Ljutica Bogdana (Kraliévitch Marko et Lioutitsa Bogdan) et Hi, hop, konjiček v kalop (Au galop), la première à cause de son thème, tiré de la poésie populaire épique serbe, et la deuxième pour sa ressemblance avec la poésie de Dehmel.

19 Paru dans Ljubljanski zvon (La Cloche de Ljubljana) en 1927 et ensuite dans l'anthologie Naša beseda (Notre parole).

20 Župančičevo literarno delo. Bibliografska skica - sestavil J.Š. (L'oeuvre littéraire de Župančič. Esquisse de bibliographie - rédigé par J.Š.) Jubilejni zbornik, str. 115 (Hommage à Župančič, p. 115) 
En 1915, son premier enfant vint au monde et le poète, heureux de cet événements, et pour chasser les pensées tristes causées par les horreurs de la guerre, publia deux recueils pour enfants: Sto ugank (Cent énigmes) et Ciciban (Tsitsiban). L'auteur de cette monographie les cite dans l'ordre inverse puisque Sto ugank (Cent énigmes) parurent les premières, en septembre, tandis que Ciciban ne parut qu'au mois de décembre. Voilà pourquoi l'affirmation de Tesnière que le recueil Sto ugank (Cent énigmes) ne parut que quelques mois après Ciciban, n'est pas exacte.

A propos de Ciciban (Tsitsiban), Tesnière écrit qu'un des secrets de la création de Župančič, c'est "de savoir allier, avec une grande justesse d'oreille et de sentiment, le rythme et le son des mots et leur sens... dans une langue musicale comme le slovène, il est possible à tirer de là des effets ravissants, malheureusement difficiles à rendre en français." 21 Tesnière constate bien que la forme des poésies de Ciciban (Tsitsiban) est constituée des onomatopées et d'un rythme particulier, d'une succession d'impressions, de dialogues animés, de personnifications tandis que le contenu est composé de thèmes simples ou philosophiques. Tesnière aurait pu élargir le choix des poésies de ce recueill, en prenant encore par exemple Uspavanka (Kaj bo sinku sèn prineslo?) (La berceuses. Qu'est-ce qui apportera le sommeil au fiston?), Breza in hrats (Le bouleau et le chêne), Pomladna ladja (La barque de printemps), Mehurčki (Les bulles de savons).

Tesnière affirmait que le recueil Sto ugank (Cent énigmes) était adressé aux enfants et aux simples paysans qui éprouvent une joie naïve à les résoudre. De nombreuses énigmes de Župančič s'inspirent il est vrai du monde matériel des paysans et de la campagne mais elles s'adressent aussi bien aux adolescents qu'aux personnes éveillées de quelle que soit leur classe, aussi bien aux intellectuels qu' aux paysans. Le poète y emploie en effet des comparaisons et des métaphores souvent assez compliquées et exigeantes.

Pour conclure le choix des quatre recueils de poésies pour enfants, l'auteur de la monographie sur Župančič trouva convenable d'y ajouter Velikonočno epistolo sinu (Epître pascale à mon fils) qu'il trouva réimprimée dans l'anthologie de Naša besed'a (Notre verbe). A la fin du chapitre, l'auteur répète qu'on aurait tort de considérer avec mépris ou comme inférieur d'un point de vue esthétique la poésie lyrique pour enfants de Župančič.

Voyons un peu la traduction des poésies mentionnées par Tesnière. Dans Pisanice (Les oeufs de Pâques) il traduit merveilleusement des expressions dialectales comme čižmice (les petits souliers), čemer (la ceinture), devojka (la jeune fille) et valje (tout de suite). La traduction de ce recueil correspond parfaitement du point de vue contenu mais les rimes, elles, pour la plupart, manquent à quelques exceptions près. Les poésies $H$ i, hop, konjiček v kalop! (Au galop) et Lahkih nog naokrog (D'un pied léger dansons 
en rond) perdent leurs rimes dans la traduction française, se vidant aussi de leur sens, de leur message, de leur but.

Dans la poésie Zvonovi (Les cloches) du recueil Ciciban (Tsitsiban) par contre, Tesnière fit beaucoup d'efforts pour reproduire la mélodie de l'original mais s'en éloignant souvent du point de vue sémantique. Dans la traduction, assez fidèle de Zeleni Jurij (Saint Georges le Verdoyant) Tesnière a apparemment mal compris le vers: že za vodó čez travnike jaše - déjà sur son cheval il va quérir de l'eau; "za vodo" signifie ici "pres de l'eau, du ruisseau, de la rivière" et non pas "il va quérir de l'eau, il va prendre de l'eau" qui n'a pas de sens dans ce contexte.

La traduction de la poésie Ciciban in čebela (Tsitsiban et l'abeille) est satisfaisante du point de vue contenu, plus ou moins satisfaisante du point de vue mélodie (rimes) mais à certains endroits elle s'éloigne hyperboliquement du contenu de l'original qui est lui beaucoup plus fin: Jokala brez potrebe/ bi šele mama moja-Attends voir comme ma maman / versera des larmes à torrents; a očka mu nabije/ s svetlično bilko zadnjo stran - mais papa lui accomodel la derrière à coup de botte; de plus, l'expression de Tesnière un bon garçon pour l'expression imagée populaire fant od fare employée par Župančič, est un équivalent assez médiocre. Les autres poésies, telles que Medved $z$ medom (L'ours et le miel), Naše luči (Nos lumières) connaissent une bonne traduction tandis que Lenka et Turek (Le Turc) étant sans rimes ne se lisent pas aussi bien qu'en slovène car la sonorité y fait défaut; dans la poésie Turek (Le Turc), dans le vers Ali fes, turški fes - Ou un fez, un fez de Turc, la conjonction ali ne correspond pas au sens de la conjonction ou employée par Tesnière, mais signifie mais.

L'auteur de la monographie sur Župančič fit un bon choix dans le recueil de Sto ugank (Cent énigmes): le choix est excellent tout comme la traduction des treize énigmes. Parmis les traductions particulièrement réussies on peut citer celles de Reka (La rivière) et de Brzojav (Le télégraphe). Il réussit à capter et à reproduire le rythme des deux poésies à l'aide des rimes sonores. La rivière montre de nouveau toute l'imagination et l'invention de Tesnière dans l'expression:

$$
\begin{aligned}
& \text { Mladinka-planinka } \\
& \text { ženka-dolinka } \\
& \text { starka-primorka }
\end{aligned}
$$

$$
\begin{aligned}
& \text { Jeune gaillarde - montagnarde } \\
& \text { vers la trentaine - habite la plaine }
\end{aligned}
$$$$
\text { vieille commère - au bord de la mer. }
$$

L'original de Župančič, il est vrai, est extrêmement condensé tandis que la version de Tesnière est plutôt élargie et descriptive.

On peut dans la traduction de la poésie Velikonočna epistola sinu (Epître pascale à mon fils), relever quelques curiosités. Župančič aime beaucoup utiliser les verbes ce qui est dans l'esprit de la langue slovène, tandis que Tesnière en français emploie aussi souvent des noms: važno je samo ...,.] kaj riše zarja zjutraj na nebo,/kateri grm je še brstjè pognal,/ in kje škrjanček gnezdi, kos, strnad. - [il n'y a d'important/ que ... ,] les dessins de l'aube au ciel matutinal,/ et le bourgeonnement des germes sur les branches, et les nids du bruant, du merle et de l'alouette. Les vers suivants sont 
traduits assez librement en ce qui concerne le contenu, la liste des jouets de l'enfant se différencie beaucoup en comparant la traduction et l'original: In važen je tvoj skiro, tvoja žoga,/ in če so v redu tvoje baterije,/ tvoj matador... Il n'y a d'important que ton polichinelle,/ ton matador, ta balle et tes soldats de plomb. On comprend plus difficilement que Tesnière en traduisant le vers Važen je južni, severni tečaj ait utilisé le mot le courant à la place du mot le pôle, peut-être l'a-t-il fait pour favoriser la rime de important- courant?

Après avoir passé en revue, noté et apprécié les divers domaines de la création littéraire de Župančič et après avoir réussi à les présenter dans ses traductions, Tesnière écrivit le chapitre Youpantchitch traducteur où appuyé sur les sources existantes, Anton Debeljak $^{22}$ et autres, il donna un court aperçu et les caractéristiques de l'activité de Župančič dans le domaine de la traduction. Très laborieux, Župančič traduisit en slovène jusqu'alors plus de 30 ouvrages de 6 langues européennes dufférentes. Plus de la moitié de ces traductions sont faites de l'anglais (Shakespeare, Shaw, Dickens, Chesterton etc.); il y a une grande différence de qualité entre ses premières traductions (Jules César et Le Marchand de Venise 1904, 1905) et les remaniements intégraux et refontes totales $(1922,1921)$. Le poète décida de présenter aux Slovènes l'oeuvre intégrale de cet auteur dramatique anglais la traduisant systématiquement, et avec grande maîtrise. Une place importante est occupée par les traductions en slovène d'auteurs français comme Maupassant (déjà en 1912), Daudet, Flaubert, Anatole France, Rostand, Volatire et Mérimée; la traduction slovène de Cyrano (E. Rostand) espiègle et spirituelle est très réussie. Il traduisait relativement peu de textes allemands, Marie Stuart de Schiller fait figure d'exception, car à cette époque-là, les intellectuels slovènes lisaient encore la littérature allemande dans la langue d'origine. Il faut mentionner la traduction de "Juge de Zalaméa" de Calderon pour littérature espagnole (en 1912) et de deux chants de la Divine Comédie de Dante pour la littérature italienne. Il se chargea également de nombreuses traductions pour le Théâtre provincial d'avant-guerre et du Théâtre National d'après-guerre où il était engagé comme "dramaturge". Par ses traductions d'oeuvres occidentales, il ne cessait d'enrichir la langue slovène lui donnant beaucoup de souplesse, car s'il n'avait pas maîtrisé les lois et les procédés de sa propre langue, il n'aurait pas pu traduire toutes ces oeuvres avec tant de succès. Župančič-traducteur ne se perdait point en descriptions et élargissements du texte mais était toujours soucieux de trouver le mot juste soit dans la langue littéraire soit dans le dialecte de son pays, sinon il avait recours aux néologismes.

Dans le dernier chapitre de la monographie, Le style de Youpantchitch, Tesnière développe assez longuement les pensées suivantes: Župančič crée toujours spontanément, sans contraintes. Issu de la poésie populaire, il veut rester proche du peuple. C'est pourquoi sa poésie n'est pas aristocratique (bien qu'elle le fût assez dans

22 A. Debeljak: Oton Župančič - prevajalec (Oton Župančič - traducteur). Jubilejni zbornik, str. 99-106 (Hommage Župančič, pp. 99-106). 
sa jeunesse). Il voudrait que sa poésie peigne le monde et la vie d'une manière réaliste et objective tout en restant variée dans ses thèmes. Ainsi que tous les petits peuples les Slovènes, eux non plus, ne sentent en géneral pas le besoin de s'exprimer en langue cultivée, trop affairés à lutter pour leur pain quotidien. Župančič, au contraire, est extrêmenent sensible à la langue, à son originalité, à sa fidelité, souplesse et beauté, ce qu'il prouva en tant que dramaturge (dans le conflit sur la prononciation de la lettre $l$ ), poète et traducteur. Župančič a un génie inné de la langue mais il est à la fois travailleur, soucieux et critique. Avec lui en tant qu'artiste, la langue slovène atteint une puissance expressive, métaphorique et suggestive ecxeptionnelle. "Il sent la force des mots, connaît l' art de les mettre en valeur par des alliances heureuses, et sa langue atteint ainsi à une puissance d'évocation et à une intensité expressive qui sont uniques en slovène et qui plongent ses compatriotes dans l'admiration" ${ }^{23}$ Le slovène est une langue mélodieuse, riche surtout en voyelles et Župančič, maître de la musique, en sut faire bon usage dans sa langue maternelle. Il est sans égal dans la formation du vers et du rythme ne suivant point les règles d'un art poétique classiciste mais seulement le sujet donné, son sentiment et son goût (l'article "Rythme et mètre"). Il n'a pas nécessairement besoin de rimes, parfois, il se contente de l'assonance mais générelament, il a une attirance pour le vers libre (Župančič possède plus de poésies en strophes et rimées que Tesnière ne le croie). Dans ce chapitre intéressant, Tesnière parle du caractère de la personnalité et de la création de Župančič ainsi que de ses idées sur la langue poétique et le vers s'appuyant sur la fameuse interview d'Izidor Cankar avec le poète. ${ }^{24}$ Mais on s'attendait à trouver, dans ce chapitre, "Le style de Youpantchitch", une analyse critique et concrète de sa poésie lyrique, la description de l'évolution et de l'entralecement des styles de recueil en recueil: le style de la poésie populaire, celui de la décadence, de l'impressionisme, du symbolisme et l'expressionnisme, c'est cette évolution dans le style qui fait défaut dans ce chapitre qui par ailleurs est excellent.

La Conclusion est un chapitre court où l'auteur parle de la perception du poète. Légèrement en contradiction avec le chapitre précédent, où il affirmait que Župančič était populaire et réaliste, il dit ici qu'il est guidé dans sa création par son intuition et par là d'un accès difficile au large public. Ses compatriotes, tout en reconnaissant en lui leur grand poète contemporain, ne voient pas en lui le plus grand poète, "plus grand même que le divin Prešeren". La plupart des critiques slovènes ne voyait en lui que le poète tandis que, selon l'opinion du poète- même, la critique des catholiques était, elle, trop partiale; ${ }^{25}$ ces critiques n'empêchèrent point la jeune génération de prendre

L. Tesnière: O. Joupantchitch, p. 357/358.

Izidor Cankar: Iz življenja in delovanja naših umetnikov (Sur l'oeuvre et la vie de nos artistes). II. Oton Župančič, Dom in svet (La patrie et le monde), 1911, pp. 75-77; isti (du même auteur: Obiski (Les visites), Nova založba 1920, pp. 165-176.

La déclaration que Župančič a faite à Izidor Cankar dans l'interview citée ci-dessus. Le rapport de la critique catholique envers le poète a essentiellement changé d'après ce que Finžgar écrit dans la 
Župančič pour maître et pour modèle. Župančič est encore peu connu en dehors de la Slovénie: Tesnière cite dans l'ordre chronologique les traductions de quelques poésies en différentes langues, les articles écrits sur le poètes et le livre d'Arturo Cronia. "La tragédie d'Oton Youpantchitch est celle des petites langues. La notoriéte mondiale d'un auteur est en quelque sorte le produit de sa notoriété nationale par le coefficient de diffusion de la langue qu'il emploie... D'autre part, il est difficile de traduire un poète. On a beau faire, une traduction n'est jamais qu'une retraite stratégique, dont la seule ambition doit être de perdre le moins possible des beautés de l'original. ${ }^{26}$ De même que Prešeren, Župančič lui-aussi en tant que membre d'un petit peuple et, quoique grand poète, il est presque impossible à traduire, ne pourra jamais occuper place à laquelle il aurait droit dans la littérature mondiale. C'est à cause de cette situation que Tesnière voudrait par son livre vraiement faire connaître Župančič à ses compatriotes français. ${ }^{27}$

L'auteur ajoute à son oeuvre une Notice bibliographique. Il y cite les sources dont il se servit critiquant de nouveau non sans éloges le livre de Cronia (1928). Malheureusement, on ne trouve pas parmi les sources la première étude importnate sur Župančič, à savoir celle de Niko Bartulović qui parut déjà en 1924 dans Srpski književni glasnik (Le Messager littéraire serbe). ${ }^{28}$

Dans la dédicace bilingue au poète se trouvant au début du livre, Tesnière se qualifie lui-même de traducteur Juda, traître, se rendant bien compte qu'il ne pourra jamais transmettre parfaitement l'art exigeant de Župančič. Lors de la parution du livre, il envoya au poète un exemplaire richement relié qui se trouve de nos jours dans la pièce commémorative du Musée municipal de Ljubljana. Josip Vidmar nous fait savoir que le poète en voulut à Tesnière parce qu'il osa le comparer à Prešeren et qu'il arrêta assez vite la lecture de son livre. ${ }^{29}$ On pourrait en déduire aussi en lisant l'introduction à cette monographie que Župančič n' aimât pas l'analyse des sources étrangères de sa poésie et la citation des parallèles étrangères de Tesnière.

Et comment le livre de Tesnière fut-il reçu du public slovène et quel fut son accueil dans le monde? Dans le journal Ljubljanski zvon (La Cloche de Lioubliana) le romaniste Jožko Prezelj ${ }^{30}$ cita quelques données biographiques sur l'auteur: en

critique des premiers deux recueils parue dans Dom in svet (Patrie et le monde) 1911, p. 402.

L. Tesnière: O. Joupantchitch, p. 370.

27 La situation de Župančič dans le mode est bien meilleure, puisque à partir des années soixantes surtout, on trouve ses oeuvres dans les anthologies accompagnées du commentaires et traduites dlans les langues étrangères non seulement par les Italiens et les Français mais aussi par les Russes, Tchèques, Bulgares, Anglais, Macédoniens, Letons, Hongrois et les Russes Blancs, Croates et plusieurs fois par les Serbes.

28 N. Bartulović: Oton Župančič. SKG 1924, livre 11 (janvier-avril), livre 12 (mai-août).

29 J. Vidmar: Iz dnevnika (Le journal). Delo (le Travail), 5. II. 1966. - Du même auteur: Obrazi (Les portraits), DZS, p. 247. 
rédigeant le livre, il était professeur des langues slaves à l'Université de Strasbourg et donna durant l'année académique 1927/28 des cours de littérature slovène commençant avec Trubar, passant par Prešeren pour en arriver à Župančič. Ensuite, l'auteur de l'article résume le contenu du livre y compris la partie monographique et les traductions suivant l'évolution du poète et la chronologie des recueils. Il dit que l'auteur avait choisi d'analyser les exemples et les traductions les plus illustratives et les plus intéressantes pour les Français et que ses traductions étaient faites consciencieusement et avec sentiment mais restent souvent sans rimes. Pour terminer, il souligne son rôle d'intermédiaire entre les deux cultures et l'importance du livre, en remerciant vivement son auteur pour cette contribution.

Dans la revue catholique Čas (Le Temps), la critique du livre fut écrite par Frst., l'abréviation du critique d'art, l'historien France Stele ${ }^{31}$ Tesnière relia la vie de Župančič avec son oeuvre, il sut saisir correctement son art, dans ses traductions, il suivait le rythme du texte original omettant néanmoins les rimes. Le livre présente d'une manière magnifique le poète et les Slovènes au reste du monde mais on regrette que les traductions soient libres et sans vraie beauté poétique. Stelè critique quelques passages du livre offensant par son style politique et libéral le caractère sérieux de l'eouvre de Tesnière. Il reproche à l'auteur d'écrire les noms slovènes en orthographe française. Dans sa conclusion, il voudrait que les Slovènes entreprennent, eux aussi, l'élaboration d'une monographie du poète.

Rajko Ložar, historien et éthnologue publia dans une autre revue d'orientation catholique, Dom in svet, une appréciation encore plus critique: ${ }^{32}$ les traductions de Tesnière n'ont aucune valeur artistique ni des prétentions de l'avoir. L'auteur choisit soigneusement le matériel dans l'histoire littéraire et le distribue logiquement. Il ajoute que Tesnière donne un bon aperçu des événements politiques concernant les Slovènes, condamnant à juste titre l'indifférence des alliés occidentaux. L'analyse de Tesnière serait plutôt psychologique, donnant moins d'importance aux idées, il parlerait en fait plus de la création que du style. Ložar lui-aussi reproche à l'auteur de se mêler des conflits slovènes et de prendre part aux attaques dirigées contre les catholiques. Il attribue à Tesnière l'opinion que c'est aussi la faute à Župančič et à sa manière de s'exprimer en slovène s'il n'est pas connu dans le monde. Le fait de comparer Župančič avec les poètes étrangers ne ferait, selon Ložar, que mieux éclairer la typologie de la Moderne slovène et non la généalogie du poète lui-même.

En rentrant de Paris, Anton Ocvirk, jeune professeur de littérature comparée et de théorie littéraire, s'arrêta, sur son chemin vers Ljubljana, à Strasbourg. Il alla voir Tesnière pour parler avec lui de la littérature et de la linguistique slovènes. Le

31 Čas (Le Temps) 1931/32, pp. 336-339.

DS 1932, pp. 242-245. 
professeur laissa à ses soins de nombreuses critiques et rapports sur son livre qui avaient paru surtout dans les périodiques français, italiens, russes, tchèques et croates. Ocvirk ne tarda pas à en publier des résumés munis des citations dans Ljubljanski zvon (La Cloche de Lioubliana) ${ }^{33}$ Ce fut de nouveau l'Italien Antonio Cronia, qui reprocha à Tesnière d'être un compilateur pédant qui ne sachant pas s'identifier au poète et ne pouvant pas ainsi donner de nouveaux résultats. Parmi les Croates, ce fut Ivo Hergešić qui déclara que les traductions de Tesnière étaient sans charme poétique, ne correspondant au texte original et que la partie dans la monographie traitant de littérature comparée aurait dû être plus accentuée. Le livre sur Župančič attira aussi l'attention du Tchèque Oton Berkopec, admirateur de poète.

La deuxième anthologie française de la poésie lyrique de Župančič parut presque cinquante ans après la première, étant en comparaison avec celle-ci plus mince, ne contenant que 23 poésies quoique renfermant les plus récentes. ${ }^{34}$ Le choix y est assez différent: le recueil $\check{C}$ aša opojnosti (Une coupe d'ivresse) n'y figure pas, $\breve{C} e z$ plan (A travers la plaine) y est représenté par quatre poésies, Samogovori (Les Monologues) par six poésies, $V$ zarje Vidove (Aux aurores de la Saint-Guy) par sept poésies, Ciciban (Tsitsiban) par deux et Zimzelen pod snegom (La pervenche sous la neige) par quatre poésies nouvelles. C'est le résultat de la collaboration de deux personnes qui ont divisé ce travail en deux parties: Viktor Jesenik, ancien émigré et étudiant slovène en France, fit une traduction fidèle en prose, le poète et essayiste français Marc Alyn la transforma en vers. L'avantage de cette deuxième anthologie consiste en une plus grande mélodiosité: les vers se terminent en rimes quoique distribuées d'une manière différente, on y rencontre même des rimes intérieures créées par la musique des voyelles et consonantes expressives. Une des qualités de cette petite anthologie, c'est la recherche passionnée et raffinée de l'expression lyrique, bref, elle diffère de la première par un plus grand sentiment poétique que Marc Alyn essaie d'obtenir en élargissant et même en changeant le texte, traduisant ainsi souvent trop librement. Parmi les meilleures traductions, les plus intéressantes, on trouve les poésies du recueil $V$ zarje Vidove (Aux aurores de la Saint-Guy): Vihar (L'orage), Slap (La cascade), Dies Irae et Glad (La faim) où la mélodie est très bien exprimée. Malgré une plus grande force d'expression et une plus grande suggestivité poétiques chez Alyn, qui se permet souvent une trop grande licentias poeticas vel traductionis, les traductions de Tesnière, elles, ne sont pas seulement plus nombreuses mais aussi plus exactes: elles transmettent au lecteur non-slovène Župančič plus directement et plus naturellement, il reproduit mieux le sujet, l'atmosphère, la pensée et le style du poète.

33 A. Ocvirk: Lucien Tesnière in kritike o njegovi knjigi "Oton Župančič". (Lucien Tesnière et les critiques de son livre "Oton Župančič"). LZ 1933, pp. 552-557, 677-681.

34 Oton Joupantchitch et Marc Alyn. Collection Poètes actuels. Association Formes et langages. Marguerittes, Gard 1978. 


\section{TESNIĖRJEVA OBRAVNAVA IN PREVAJANJE ŽUPANČIČA}

Svoje razpravjanje o Lucienu Tesnièru kot razlagalcu in posredovalcu Župančičeve pretežno lirične umetnosti bi mogli strniti $v$ naslednja sklepna spoznanja in uogotovitve:

Tesnière je svojo knjigo Oton Joupantchitch, poète slovène. L'homme et l'eouvre. Paris 1931, zasnoval in izdelal jasno in pregledno: $v$ monografskem delu spremlja pesnikovo življenje in ustvarjanje po zbirkah in področjih; poleg lirike obravnava epskega Jeralo, tragedijo Veronika Deseniška in pesmi za otroke; literarnozgodovinski pregled Župančičevega ustvarjanja vsakokrat ilustrira s številniomi prevodi izbranih pesmi in odlomkov; opušča edinole pesnikovo kratko prozo in raznovrstno esejistiko, ker sta tedaj pač še bili raztreseni po revijah; spregovori tudi o Župančiču kot prevajalcu, o njegovem ustvarjanju in razmerju do jezika ter o njegovi percepciji v javnosti.

Tesnièrova tematična in idejna oznaka glavnih pesnikovih zbirk je na splošno pravilna, deloma pa tudi subjektivna: Čašo opojnosti pojmuje nekoliko preveč hedonistično; vitalistično Čez plan označuje za liriko bolesti; v Samogovorih in v zbirki V zarje Vidove pa ne zaznava spiritualistično doživetega panteizma. Odločno premalo se ukvarja z oblikovno izraznimi vprašanji Župančičevega ustvarjanja, s formo, kompozicijo, verzom in slogom njegovih pesmi. $\mathrm{V}$ monografskih partijah knjige razodeva dobro poznavanje pesnikovega življenja ter takratnih slovenskih kulturno političnih razmer, naletimo pa tudi na nekaj literarno zgodovinskih, geografskih in etnografskih napak v stvareh, ki tedaj še niso bile strokovno raziskane ali jih kot tujec ni mogel v celoti poznati.

Kar zadeva prevajanje Župančiča, ga Tesnière v semantičnem pogledu posreduje skoraj zgledno, saj slovensko besedišče in njegove pomene na splošno obvlada, celó do manj znanih odtenkov. Spodleti mu tam pa tam pri redkejših izrazih, pomenskih dvvojčkih ali eliptičnih stavkih, razumljivo je tudi, da ne more poznati vseh slovenskih ljudskih rekel. Nadalje je značilno, da je $\mathrm{v}$ primerjavi s pesnikovim neposrednim, zgoščenim in lahkotnim izražanjem pri prevajanju pogosto opisen, daljši, tudi okoren, na drugi strani pa v svoji elegantni francoščini sposoben številnih domislenih besednih iger.

Ena izmed najbolj značilnih lastnosti Župančičeve lirike je njena muzikalnost, z njo pesnik sugestivno slika in posreduje določeno razpoloženje $v$ naravi in v sebi. Tu Tesnière skoraj docela odpoveduje, saj mu redkokje uspe prenesti v prevod bogato zvočnost pesnikovih zunanjih in notranjih rim ter ekspresivnih samoglasnikov in soglasnikov. Toda vsebinska stran besedila, čustvo in misel, pa tudi slog in ritem so $v$ njegovih prevodih redno skrbno, ustrezno in učinkovito podani. Zato Tesnièrovi prevodi Župančiča bolj avtentično posredujejo kakor muzikalno in poetično bolj uspeli. toda vsebinsko kar preveč svobodni in digresivni Alynovi.

Če pomislimo, da je bil prvenstveno jezikoslovec in da je med nami živel le nekaj let, so Tesnièrova litrerarnozgodovinska in prevajalska prizadevanja in dosežki v knjigi o Otonu Župančiču vredni vsega našega spoštovanja, občudovanja in hvaležnosti. 